\title{
Formation of nano-voids in transparent dielectrics by femtosecond lasers
}

\author{
Eugene G. Gamaly ${ }^{\mathrm{a}, *}$, Saulius Juodkazis ${ }^{\mathrm{b}}$, Hiroaki Misawa ${ }^{\mathrm{b}}$, Barry Luther-Davies ${ }^{\mathrm{a}}$, \\ Andrei V. Rode ${ }^{a}$, Ludovic Hallo ${ }^{c}$, Philippe Nicolai ${ }^{c}$, Vladimir T. Tikhonchuk ${ }^{\mathrm{c}}$ \\ ${ }^{a}$ Laser Physics Centre, Research School of Physical Sciences and Engineering, The Australian National University, Canberra ACT 0200, Australia \\ ${ }^{\mathrm{b}}$ Research Institute for Electronic Science, Hokkaido University, CRIS Building, Sapporo 001-0021, Japan \\ ${ }^{\mathrm{c}}$ Centre Lasers Intenses et Applications, UMR 5107 CEA, CNRS, Université Bordeaux 1, 33405 Talence Cedex, France
}

Available online 1 November 2007

\begin{abstract}
Formation of three-dimensional (3D) patterns of $0.2-0.5 \mu \mathrm{m}$ diameter voids inside a transparent solid has been demonstrated. The nano-voids could be created with a spacing $\sim 2 \mu \mathrm{m}$ thus suggesting the possibility of permanent $3 \mathrm{D}$ optical memory with density $\sim 100 \mathrm{Gbit} / \mathrm{cm}^{3}$. Extremely high pressures of $\sim 10 \mathrm{TPa}$ and temperatures up to $10^{5} \mathrm{~K}$ can be produced by a single sub-picosecond laser pulse tightly focused inside transparent dielectrics. Analysis of the size of the void and the shock affected zone as a function of the deposited energy shows that the experimental results can be explicitly understood on the basis of conservation laws and be modeled by plasma hydrodynamics. Therefore, the size of the void can be controlled and predicted by proper focusing conditions and laser parameters.
\end{abstract}

(C) 2007 Elsevier B.V. All rights reserved.

PACS: 81.07.-b; 96.50.Fm; 62.50.+p; 81.40.-z; 47.40.Nm

Keywords: Femtosecond laser microfabrication; Shock waves; Dielectric breakdown; Sapphire; Nano-structuring

\section{Introduction}

The pressures in excess of $0.1 \mathrm{TPa}$ have been obtained using a diamond anvil in stationary conditions, whilst transient pressures behind shock waves generated by chemical or nuclear explosions or generated using powerful lasers up to $50 \mathrm{TPa}$ have been reported [1]. Recent studies have demonstrated [2-5] that sub-picosecond laser pulses tightly focused inside transparent dielectrics can produce detectable sub-micrometer-sized structural modifications, including voids. This requires intensities in excess of $I=0.1 \mathrm{PW} / \mathrm{cm}^{2}$, which results in a highly non-linear light-matter interaction with most dielectrics being ionised early in the laser pulse. So far the forma-

\footnotetext{
* Corresponding author.

E-mail address: gam111@rsphyse.anu.edu.au (E.G. Gamaly).
}

tion of voids in silica was associated with self-focusing of the laser beam [2].

Here we present, experimental evidence that one can create TPa pressures, many times exceeding the strength of any material, with low energy pulses from a conventional tabletop sub-picosecond laser without self-focusing. We demonstrate that nano-voids are formed by the extreme temperatures and pressures created by optical breakdown and these drive shock and rarefaction waves. The nano-void can be used in three-dimensional (3D) memory applications.

\section{Experimental}

The interaction between an intense laser pulse and the material is fundamentally different when the laser beam is tightly focused inside a transparent solid rather than on its surface. Inside the solid the interaction zone containing 
high energy density is confined in a cold and dense material. For this reason hydrodynamic expansion is highly restricted and proceeds as a micro-explosion when a high pressure and temperature volume is created inside bulk (Fig. 1). In our experiments $100 \mathrm{~nJ}, 150 \mathrm{fs}$, laser pulses at $800 \mathrm{~nm}$ were tightly focused using high numerical aperture objective lens $(\mathrm{NA}=n \times \sin (\theta)=1.35$, where $\theta$ is halfangle of focusing cone) inside a dielectric.

To avoid self-focussing, and thus deliver the laser energy to a specific location inside the transparent solid, requires the laser power to be lower than the critical value for self-focusing. This critical power $P_{\text {cr }}$ depends on the nonlinear part of the refractive index $n_{2}=n_{0}+n_{2} I$ as $P_{\text {cr }}=\lambda^{2} /\left(2 \pi n_{0} n_{2}\right)[6]$. The pulse energy was varied in a range $20-150 \mathrm{~nJ}$. This irradiance exceeded the optical breakdown threshold but the pulse power was below $P_{\text {cr }}$. We define the focal volume as that confined inside a cylinder with the diameter equals to FWHM of laser intensity. The radius of this cylinder for a Gaussian beam is $r \cong$ $\sqrt{\ln 2 / 2} r_{0}$, while $r_{0}=0.61 \lambda /$ NA. The axial length of the focal volume is twice the Rayleigh length $2 z_{0}=2 \pi r_{0}^{2} n / \lambda$. Hence, the focal volume is $V \simeq 0.95 \lambda^{3} n / \mathrm{NA}^{4}$ and the focal spot area is $S \simeq 0.41 \lambda^{2} /(\mathrm{NA})^{2}$. For our experiments: $V=0.29 \mu \mathrm{m}^{3}$ and $S=0.15 \mu \mathrm{m}^{2}$ leading to an intensity of $0.35 \mathrm{PW} / \mathrm{cm}^{2}$ for a $100 \mathrm{~nJ}$ pulse.

An array of laser-affected spots was created aligned along the $c$-plane inside the sapphire crystal using a sequence of single laser pulses. The sample was then cleaved along the $c$-direction and examined using a scanning electron microscope (SEM). The observed pattern is shown in Fig. 2. The central void was surrounded by a shell extending to about twice the void diameter. This shell was identified as amorphous material by chemical etching, since amorphous sapphire has a much higher solubility in HF acid compared with crystalline sapphire [7]. The teardrop shape along the direction of pulse propagation is due to spherical aberration caused by the refractive index mismatch between the immersion oil and sapphire [8].

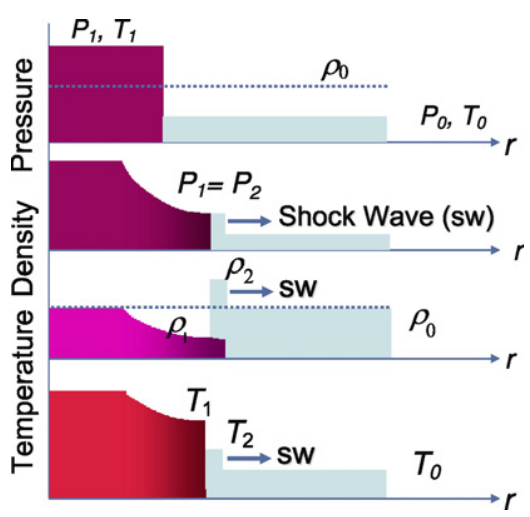

Fig. 1. Schematics of pressure, $P$, temperature, $T$, and density, $\rho$, transients in the case of strong explosion and shock wave (SW) formation (the index 0 marks the unperturbed values). The initial high pressure $P_{1}$ and temperature $T_{1}$ sphere is centered at $r=0 ; r$ denotes a radial coordinate.

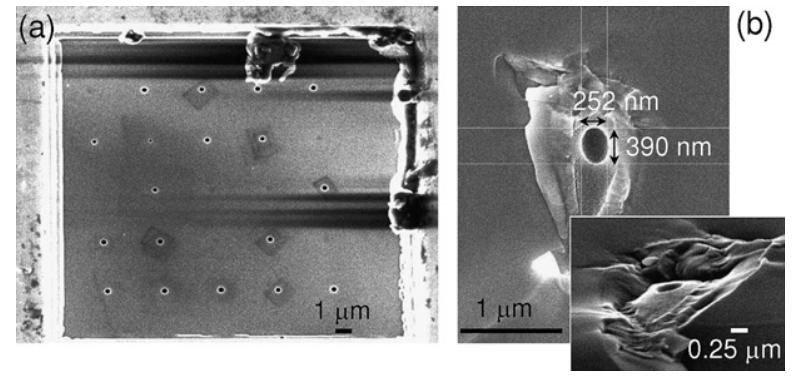

Fig. 2. (a) The top-view (c-plane) SEM image of array of voids recorded at $5 \mu \mathrm{m}$ depth in sapphire. The voids were revealed by FIB etching with $\mathrm{Ga}^{+}$ions. Pulse energy was $120 \mathrm{~nJ}$, pulse duration $150 \mathrm{fs}$ at the wavelength of $800 \mathrm{~nm}$. (b) The typical side-view (along $c$-axis) SEM image of the void surrounded by amorphous shell inside sapphire. The inset shows a tilted view of the void.

\section{Results and discussion}

\subsection{Void formation}

The formation of a void and shock-affected zone can be understood from simple reasoning based on the laws of mass and energy conservation. For simplicity, a spherically symmetric motion is considered. The shock wave propagating in a cold material loses its energy due to dissipation, that is, due to the work done against the internal pressure, the Young or bulk modulus, that resists material compression. The distance $r_{\mathrm{s}}$ at which the shock front effectively stops defines the shock-affected volume. At this point the shock wave converts into a sound wave, which propagates further into the material without inducing any permanent changes. The distance where the shock wave stops can be estimated from the condition that the internal energy at the shock front is comparable to the absorbed energy: $4 \pi r_{\mathrm{s}}^{3} \rho_{0} / 3=E_{\text {abs }}$. At this position the pressure behind the shock front equals the internal pressure of the cold material [9]. One can reasonably suggest that the sharp boundary observed between the amorphous (laser-affected) and crystalline (unaffected) sapphire corresponds to the distance where the shock wave effectively stopped.

Computer simulations were performed in spherical geometry using the hydrodynamic code Chivas [11], which considered the ionisation equilibrium, separate temperatures for electron and ion components, and electron heat conduction. The cylindrical region where the energy was absorbed was approximated by a sphere of the same volume. The absorbed energy $E_{\text {abs }}=50 \mathrm{~nJ}$ was deposited homogeneously in this spherical volume with radius $r_{\mathrm{d}}=0.13 \mu \mathrm{m}$ in a material with initial density $\rho_{0}$. The equation of state implemented in the code [11] describes solidmelt-plasma and reverse transitions. The simulations started after the end of the laser pulse with the initial electron temperature $T_{\mathrm{e}}=26.2 \mathrm{eV}$ and the average ion charge (number of electrons that are stripped off an atom) $\langle Z\rangle=4.3$ (see Section 3.2). The hydrodynamic motion commences after the electrons transfer the absorbed energy to ions in excess of that necessary to break the inter-atomic 

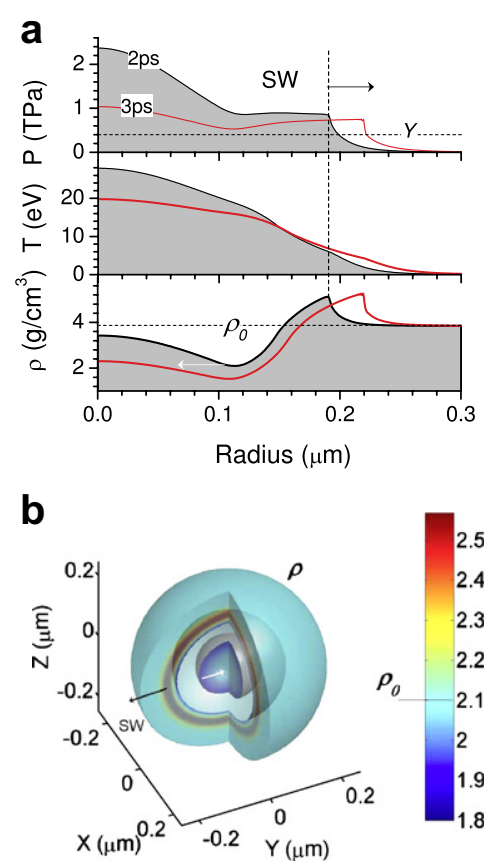

Fig. 3. (a) Spatial profiles of pressure, temperature, and mass density at 2 and $3 \mathrm{ps}$ after the laser-induced micro-explosion in sapphire [10]. The density $\rho_{0}=3.89 \mathrm{~g} / \mathrm{cm}^{3}$ and Young modulus $Y=0.4 \mathrm{TPa}$ of sapphire are shown by the dashed lines. (b) The initial moment of the hydrodynamic motion in silica glass at the time moment of $1 \mathrm{ps} ; \rho_{0}=2.1 \mathrm{~g} / \mathrm{cm}^{3}$. Calculations by code Chivas [11].

bonds. The strong shock wave emerges at the outer surface of sphere where the energy was deposited compressing the material to a density twice its initial value. Subsequently, the pressure behind the shock front rapidly decreases with distance, and finally the shock transforms into an acoustic wave. A void is formed behind the shock front and its radius gradually increases (see Fig. 3). The compression ratio at $1 \mathrm{~ns}$ reaches its asymptotic value of $\delta=\rho / \rho_{0}=$ 1.1 , which qualitatively agrees with the density of the amorphous layer deduced from the experiments, $\delta_{\exp }=1.14$. We note that the remaining gas density in the central void region at $1 \mathrm{~ns}$ is above $0.02 \mathrm{~g} / \mathrm{cm}^{3}$. After the pressure release, the vapor should be condensed on the cavity walls.

\subsection{Ionisation and absorption}

The multi-photon and avalanche ionisations are responsible for the optical breakdown in dielectrics [12-15]. Number of photons required to transfer an electron from the valence to the conduction band is $n_{\mathrm{ph}}=\Delta_{\mathrm{g}} / \hbar \omega$, here $\Delta_{\mathrm{g}}$ is the band gap. The six-photon ionisation rate in sapphire is a fast process $[16,17]: w_{\mathrm{mpi}} \cong \omega n_{\mathrm{ph}}^{3 / 2}\left(\varepsilon_{\mathrm{osc}} / 2 \Delta_{\mathrm{g}}\right)_{\mathrm{ph}}^{n}$ (here $\varepsilon_{\mathrm{osc}}$ is the oscillation energy of electrons in the laser field and $\omega$ is the laser frequency).

The ionisation rate in dielectrics can be expressed through the effective electron collision frequency [16]: $w_{\mathrm{a}} \cong\left(\varepsilon_{\mathrm{osc}} / \Delta_{\mathrm{g}}\right) \omega^{2} v_{\text {eff }} /\left(v_{\text {eff }}^{2}+\omega^{2}\right)$. The process of energy absorption by oscillating electrons occurs first, early in the pulse, due to electron-phonon collisions, $v_{\text {eff }} \approx$ $v_{\mathrm{e}-\mathrm{ph}} \ll \omega$, and later by electron-ion collisions $v_{\mathrm{eff}} \approx v_{\mathrm{ei}}$ $\omega_{\text {pe }}$, where $\omega_{\text {pe }}$ is the electron plasma frequency. The interplay of two processes, multi-photon and avalanche ionisation, swiftly converts the irradiated part of the crystal into a plasma with electron density $n_{\mathrm{e}} \sim 10^{23} \mathrm{~cm}^{-3}$ and average ion charge of 4-5 in only a few fs at the beginning of the laser pulse. Since the electron density exceeds the critical density for the laser frequency, the absorption increases significantly due to transformation of the dielectric into the plasma state, which leads to a sharp increase in the energy density deposited by the laser. The laser energy becomes efficiently absorbed in a volume significantly smaller than the focal volume.

The electron-ion energy exchange rate in the solid density plasma is $v_{\mathrm{T}} \sim\left(m_{\mathrm{e}} / M_{\mathrm{i}}\right) v_{\mathrm{eff}}$ and an energy equilibration time, $\sim 2 \mathrm{ps}$, is much longer than the pulse duration. The deposited energy goes into heating the electrons while the ions remain cold. The electron energy density in the absorbing region can be estimated taking ionisation losses into account as $W_{\mathrm{dep}}=2 A F_{\mathrm{p}} / l_{\mathrm{abs}}-E_{\mathrm{ion}}$, where $F_{\mathrm{p}}$ is the pulse fluence, and the second term includes the total losses to ionisation. The ionisation equilibrium is established by the end of the pulse because the recombination time is shorter than the pulse duration, and the electron density, therefore, saturates. Thus, the maximum pressure $P_{\max }$, which subsequently drives a shock wave can simply be estimated taking $E_{\mathrm{p}}=100 \mathrm{~nJ}, F_{\mathrm{p}}=70.4 \mathrm{~J} / \mathrm{cm}^{2} ; S=0.15 \mu \mathrm{m}^{2}$, $l_{\mathrm{abs}}=65 \mathrm{~nm} ; A=0.61 \quad$ and $\quad V_{\mathrm{abs}}=10^{-2} \mu \mathrm{m}^{3}, \quad P_{\max }=$ 13.4 TPa. This pressure significantly exceeds the Young's modulus of sapphire, $Y_{0}=0.4 \mathrm{TPa}$, thus the shock wave generated by the plasma forms the void with a surrounding dense shell.

The laser-induced micro-explosion is characterized by the total absorbed energy, initial density, and radius of the energy deposition zone, because the energy deposition time into the ions $(\sim 1 \mathrm{ps})$ is negligible compared with the characteristic hydrodynamic time $(\sim 1 \mathrm{~ns})$. The heating rate is obtained by dividing the maximum temperature by the electron-ion energy transfer rate $T_{\mathrm{e}} / v_{\mathrm{T}} \simeq 1.5 \times 10^{17} \mathrm{~K} / \mathrm{s}$. The cooling rate is defined by the ratio of the maximum temperature and the shock-wave stopping time $T_{\mathrm{e}} / 10^{-9} \simeq 3 \times 10^{14} \mathrm{~K} / \mathrm{s}$.

\section{Conclusion}

The voids and amorphised regions of sub-micrometer cross-sections were created inside sapphire by single focused fs-pulses. The mechanism of their formation has been determined as a result of micro-explosion. The micro-structures can be used in 3D high density memory applications.

\section{Acknowledgements}

Support by a Grant-in-Aid Kiban-B(2) No. 19360322 is acknowledged. We are grateful to Prof. W.Z. Krolikowski for discussions. 


\section{References}

[1] P.F. McMillan, Pressing on: the legacy of Percy W. Bridgeman, Nat Mater. 4 (2005) 715.

[2] E.N. Glezer, M. Milosavljevic, L. Huang, R.J. Finlay, T.-H. Her, J.P. Callan, E. Mazur, Three-dimensional optical storage inside transparent materials, Opt. Lett. 21 (1996) 2023-2025.

[3] C.B. Schaffer, A. Brodeur, J.F. García, E. Mazur, Micromachining bulk glass by use of femtosecond laser pulses with nanojoule energy, Opt. Lett. 26 (2) (2001) 93-95.

[4] K. Yamasaki, S. Juodkazis, M. Watanabe, H.-B. Sun, S. Matsuo, H. Misawa, Recording by micro-explosion and two-photon reading of three-dimensional optical memory in polymethyl-methacrylate films, Appl. Phys. Lett. 76 (8) (2000) 1000-1002.

[5] H. Sun, Y. Xu, S. Juodkazis, K. Sun, M. Watanabe, S. Matsuo, H. Misawa, J. Nishii, Arbitrary-lattice photonic crystals created by multiphoton microfabrication, Opt. Lett. 26 (6) (2001) 325-327.

[6] S. Akhmanov, V. Vyspoukh, A. Chirkin, Optics of Femtosecond Laser Pulses, Moscow, Nauka, 1988.

[7] S. Juodkazis, K. Nishimura, H. Misawa, T. Ebisui, R. Waki, S. Matsuo, T. Okada, Control over the state of crystallinity: sapphire, Adv. Mater. 18 (11) (2006) 1361-1364, doi:10.1002/adma.200501837.

[8] E.E. Gamaly, S. Juodkazis, K. Nishimura, H. Misawa, B. LutherDavies, L. Hallo, P. Nicolai, V. Tikhonchuk, Laser-matter interaction in a bulk of a transparent solid: confined micro-explosion and void formation, Phys. Rev. B 73 (2006) 214101.
[9] Y. Zel'dovich, Y.P. Raizer, Physics of Shock Waves and High-Temperature Hydrodynamic Phenomena, Dover, New York, 2002.

[10] S. Juodkazis, K. Nishimura, S. Tanaka, H. Misawa, E.E. Gamaly, B. Luther-Davies, L. Hallo, P. Nicolai, V. Tikhonchuk, Laser-induced microexplosion confined in the bulk of a sapphire crystal: evidence of multimegabar pressures, Phys. Rev. Lett. 96 (16) (2006) 166101.

[11] S. Jacquemot, A. Decoster, Z-scaling of collisional Ne-like X-ray lasers using exploding foils - refraction effects, Laser Part. Beams 9 (1991) 517-526.

[12] E. Yablonovitch, N. Bloembergen, Avalanche ionization and the limiting diameter of filaments induced by light pulses in transparent media, Phys. Rev. Lett. 29 (1972) 907-910.

[13] B. Stuart, M. Feit, A. Rubenchick, B.W. Shore, M.D. Perry, Laserinduced damage in dielectrics with nanosecond to picosecond pulses, Phys. Rev. Lett. 74 (1995) 2248-2251.

[14] D. von der Linde, H. Schuler, Breakdown threshold and plasma formation in femtosecond laser-solid interaction, J. Opt. Soc. Am. B 13 (1) (1996) 216-222.

[15] C. Carr, H. Radousky, A.M. Rubenchik, M. Feit, S. Demos, Localized dynamics during laser-induced damage in optical materials, Phys. Rev. Lett. 92 (2004) 087401-1.

[16] Y.P. Raizer, Laser-Induced Discharge Phenomena, Consultant Bureau, New York, 1977.

[17] L.V. Keldysh, Ionization in the field of a strong electromagnetic wave, Sov. Phys. JETP 20 (1965) 1307. 